\title{
Poor Sleep Quality Associated With High Risk Of Ventricular Tachycardia After Acute Myocardial Infarction
}

\author{
Shipeng Wang (D) ${ }^{1,2, *}$ \\ Hui $\mathrm{Gao}^{3, *}$ \\ Zewen $\mathrm{Ru}^{\mathrm{I}}$ \\ Yanan Zou' \\ Yilan $\mathrm{Li}^{\mathrm{I}}$ \\ Wei $\mathrm{Cao}^{4}$ \\ Wei Meng' \\ Jihe $\mathrm{Li}^{5}$ \\ Yuan Yao' \\ Yanxiu Zhang' \\ Xueyan Lang' \\ Yao Zhang ${ }^{1,2}$
}

'Department of Cardiology, The Second Affiliated Hospital of Harbin Medical University, Harbin 150086, People's Republic of China; ${ }^{2}$ Key Laboratory of Myocardial Ischemia, Ministry of Education, Harbin Medical University, Harbin 150086, People's Republic of China; ${ }^{3}$ Department of Cardiology, People' Hospital of Xinzheng, Xinzheng, Henan Province 45I I50, People's Republic of China; ${ }^{4}$ Department of Cardiology, Heilongjiang Provincial Hospital Affiliated to Harbin Institute of Technology, Harbin 150036, People's Republic of China; ${ }^{5}$ Department of Cardiology, The First Affiliated Hospital of Harbin Medical University, Harbin I5000I, People's Republic of China

*These authors contributed equally to this work

Correspondence: Shipeng Wang Department of Cardiology, The Second Affiliated Hospital of Harbin Medical University, Xuefu Road 246\#, Nangang District, Harbin I50086, People's Republic of China

Tel +86 I3654504286

Fax +8686296225

Email guoyunqaz@126.com
This article was published in the following Dove Press journal: Nature and Science of Sleep

Background: Sleep disorders (SDs) are usually associated with an increase in frequency of ventricular tachycardia (VT). However, the relationship between SDs and the prevalence of VT within the first week of acute myocardial infarction (AMI) remains unclear. This study aimed to evaluate their associations and potential mechanisms.

Methods: This structured questionnaire-based cross-sectional study enrolled 303 patients with AMI from a hospital in northern China. Pittsburgh Sleep Quality Index (PSQI) was used to determine sleep quality of subjects. Heart rate variability (HRV) of patients was investigated by ambulatory electrocardiography recorders. Enzyme-linked immunosorbent assay was used to measure the plasma levels of catecholamine in a subgroup including 80 patients with AMI.

Results: After adjusting to basic cardiovascular characteristics, results of multivariate logistic regression demonstrated that the global PSQI score and its main components were positively associated with VT prevalence in inpatients with AMI. There were significantly different HRV parameters interpreted as autonomic nerve activity in two groups of AMI patients with different sleep quality. In addition, we found the influence of sleep quality on plasma concentrations of adrenaline and norepinephrine in AMI patients.

Conclusion: Sleep status was significantly associated with the initiation of VT within the first week of AMI, probably due to the effect of SDs on sympathetic nerve activity. Amelioration of sleep quality and sympathetic hyperactivity may be prospective strategy to curb arrhythmias after AMI.

Keywords: ventricular tachycardia, acute myocardial infarction, sleep disorders, heart rate variability, catecholamine

\section{Introduction}

Ventricular arrhythmia is a common cause of deaths after acute myocardial infarction (AMI). Although traditional cardiac risk factors associated with an increased risk of ventricular tachycardia (VT) after AMI, such as age, hypertension, impaired systolic function, have been defined, the underlying mechanisms are not fully understood. ${ }^{1,2}$ More exploration of risk factors will help to better understand the pathological mechanisms of VTs after AMI and make preventive strategies.

It has been reported that $56 \%$ of Americans, $31 \%$ of western Europeans, $23 \%$ of Japanese, and $42 \%$ of Chinese suffered from sleep disorders (SDs). ${ }^{3}$ In a study among adult residents of Northeastern China, short sleep duration was found in approximately $53.4 \%$ of the 21,435 participants and to be associated with history of 
coronary heart disease. ${ }^{4}$ Using a multistage cluster and random sampling method, researchers reported the associations between poor sleep quality and high risk of hypertension among a representative sample of 9404 adults aged 20-93 years in China. ${ }^{5}$ Among cardiac patients, the prevalence of insomnia has been estimated to be as high as $44 \%{ }^{6}$ With increasing connections between sleep and cardiovascular disease, some have argued that SD is the 10 th potentially alterable cardiovascular risk factor. ${ }^{7}$

Evidence is mounting for links between sleep disorders and ventricular arrhythmias (VAs). A one-year follow-up study demonstrated that there is a higher incidence of ventricular extrasystoles in shift workers compared to that in daytime workers. ${ }^{8}$ Studies have suggested an association between sleep-disordered breathing and life-threatening VAs, as was demonstrated in a systematic review of 20 studies collectively evaluating $>10$ thousand people. ${ }^{9}$ Dysfunction of the autonomic nervous system may be responsible for SDs-related VT prevalence. Because SDs can contribute to cardiac sympathetic stimulation, which has been long known to exert a prominent role in genesis of VTs. ${ }^{10}$ Acute myocardial ischemia has the same effect on cardiac sympathetic nerve activity as SDs. Therefore, we hypothesize that acute myocardial ischemia accompanying AMI can further promote the SDs-induced sympathetic hyperactivity which leads to the prevalence of VT after AMI.

The aim of the present study was to investigate the association between sleep quality in a short period before AMI and the prevalence of VT in the first week of AMI. We examined sleep quality over the month prior to interview, a period during which electrophysiological changes in the heart may occur, to investigate whether short-lasting sleep disorders have an impact on the prevalence of VT following AMI. To evaluate the potential mechanisms under association between SDs with VT following AMI, we sought to reveal the potential effect of sleep disorder on heart rate variability in AMI patients. In addition, the effect of sleep quality on sympathetic nerve activity was also investigated by measuring concentrations of plasma catecholamine in patients with AMI.

\section{Materials And Methods Study Group And Design}

This cross-sectional study was approved by the Research Ethics Committee of The Second Affiliated Hospital of Harbin Medical University (ky2018-246) and all experimental procedures conformed to the ethical guidelines of the 1975 Declaration of Helsinki.

Totally, 349 patients with AMI were enrolled and signed written informed consent approved by the Ethical Committee. Twenty-five of them were precluded due to incomplete data and 21 patients were excluded according to pre-established criteria. Then, a total of 303 patients with ST-segment elevation myocardial infarction (STEMI) who underwent primary percutaneous coronary intervention (PCI) were enrolled at the Second Affiliated Hospital of Harbin Medical University in China between April 2018 and November 2018. The inclusion criteria were (1) hospitalization for characteristic chest pain lasting for $>30$ mins, admitted within $12 \mathrm{hrs}$ from the onset of above symptoms; (2) ST-segment elevation $\geq 1 \mathrm{~mm}$ in 2 contiguous electrocardiographic leads or new onset of complete left bundle-branch block; (3) primary PCI, including balloon angioplasty, thrombus aspiration, or stent implantation; (4) ischemic times between 0.5 and $12 \mathrm{hrs;}$ (5) sinus rhythm. The exclusion criteria were: (1) unconsciousness; (2) known hearing or visual impairment; (3) cardiogenic shock or persistent severe hypotension (systolic blood pressure $<80 \mathrm{~mm} \mathrm{Hg}$ ); (4) any chronic illness, such as cancer, liver cirrhosis, respiratory failure or end-stage renal failure; (5) acute cerebral infarction; history of arrhythmia or myocardial infarction; history of myocardial and valvular diseases; (6) concomitant severe ionic balance disorder, such as hypokalemia, hyponatremia, hypocalcemia, hyperkalemia; (7) known cardiac heredity ion channelopathy; (8) death during hospitalization.

\section{Clinical Variables}

All patients with AMI were admitted to the cardiac intensive care unit and transferred to the general cardiology ward 2-3 days later. Patients were evaluated by historic data collection, functional classification, Doppler echocardiography, electrocardiogram (ECG), 24-hr ambulatory electrocardiogram (Holter), and a sleep questionnaire. We conducted a complete 2D echocardiography and Doppler ultrasound examination to measure the ejection fraction (EF) and left ventricular end-diastolic internal diameter (LVEDD). Patients were assessed by ECG for at least two consecutive days after admission for location of myocardial infarction. QTc was measured by ECG machine (MAC 1200 ST, General Electric Company, USA). Medication use was ascertained by means of a standardized interview. 


\section{Ambulatory Electrocardiogram}

VT was estimated by ECG monitors in CCU and Holter recorders (pace recorder model HWM-112W, Shijiazhuang Hanwei Medical Equipment Co, Ltd) with a sampling rate of $250 \mathrm{~Hz}(4 \mathrm{~ms})$ in the general cardiology ward, respectively. Holter also was used to determine HRV as well as $24 \mathrm{hrs}$ average heart rates (AHR). VT was characterized as cardiac arrhythmia of $\geq 3$ consecutive complexes originating in the ventricles at a rate $>100 \mathrm{bpm}$. The entire study population was measured in the time domain: the standard deviation of mean $\mathrm{NN}$ (SDNN), mainly the sympathetic output; the mean of the standard deviation of all normal RR intervals for all 5-min segments (SDNNi); the standard deviation of all 5 mins mean RR intervals (SDANN); rootmean-square of successive RR interval difference (rMSSD), corresponding to parasympathetic activity; and the percentage of $>50 \mathrm{~ms}$ differences between adjacent $\mathrm{NN}$ (pNN50). Welch's averaged periodogram method was performed for frequency-domain parameters: Total frequency (TF) power $(0.000-0.400 \mathrm{~Hz})$, low frequency (LF) power $(0.040-0.150 \mathrm{~Hz})$, high frequency (HF) power $(0.150$ $0.400 \mathrm{~Hz}$ ), very low frequency (vLF) power $(0.003-$ $0.040 \mathrm{~Hz}$ ), ultra-low-frequency (ULF) power $(0.000$ $0.003 \mathrm{~Hz}$ ), LF/HF ratio.

\section{Sleep Quality Assessment}

We assessed sleep quality of the patients on the next day after admission. Sleep quality of subjects was investigated by the Pittsburgh Sleep Quality Index (PSQI). PSQI is a questionnaire that captures seven dimensions of sleep: subjective sleep quality (a subjective feeling of satisfaction in daily sleep), sleep latency (length of time for transition from full wakefulness to sleep), habitual sleep efficiency (proportion of hours slept to total hours in bed), daytime dysfunction (trouble staying awake while engaging in social activity), sleep duration and use of sleep medication, sleep disturbance (interruption of sleep). The global PSQI score and the seven dimensions listed above were assessed by the four grade system (that is, 0, 1, 2 and 3), with higher numbers reflecting worse sleep quality.

\section{Blood Sampling And Measurement Of Plasma Catecholamine Levels}

In the morning of the third day after admission, we obtained blood samples from an antecubital vein in a subgroup of 80 patients. The blood samples were stored in anticoagulated tube with ethylenediaminetetraacetic acid (EDTA). Then, the blood samples were separated via centrifugation at $3000 \mathrm{rpm}$ for $10 \mathrm{mins}$ at $4^{\circ} \mathrm{C}$. An aliquot of the EDTA plasma was stored at $-80^{\circ} \mathrm{C}$ till being assayed. According to the manufacturer's instruction, ${ }^{11}$ we measured plasma levels of adrenaline, norepinephrine and dopamine by enzyme-linked immunosorbent assay (CatCombi, IBL, Hamburg). An investigator performing these assays was blinded to the sources of the samples.

\section{Statistical Analysis}

Continuous variables were presented as mean \pm standard deviation (SD) or medians with interquartile ranges (IQR), and nominal variables were presented as frequencies and proportions. We compared baseline characteristics between participants with and without poor sleep quality using Student t-tests or non-parametric tests based on distributional properties for continuous variables and chisquare $\left(\chi^{2}\right)$ test or Fisher's exact test for nominal variables, respectively. Multivariate logistic regression analyses were conducted to estimate cross-sectional associations of selfreported sleep quality with VT following AMI. We performed analysis of multivariate logistic regression to assess the odds ratios and the corresponding 95\% confidence intervals (CIs) for the associations between VT after AMI and poor sleep quality using the global PSQI score and seven other PSQI subcomponents, adjusted for age, gender, hypertension, diabetes, history of medication, location of AMI, EF, QTc, ischemic times, LVEDD. t-tests or non-parametric tests were used to determine any significant differences in HRV and plasma level of catecholamine between two groups with or without poor sleep quality. A rank correlation analysis was performed to ascertain whether sleep quality might affect the plasma level of catecholamine among patients with AMI. SPSS 13.0 (SPSS Inc., Chicago, IL, USA) was used to perform all statistical analyses. All tests were two-sided and the level of statistical significance was set at $\mathrm{p}<0.05$.

\section{Result}

As shown in Table 1, the basic characteristics of the study sample are summarized. There were finally 195 males and 108 females enrolled in the present study. The study samples were divided into two groups according to sleep quality defined by a global PSQI score $\leqslant 5$ (good sleep quality) and a global PSQI score $\geqslant 6$ (poor sleep quality). Among 303 study participants (64.4\% male; mean age: 59.60 years; SD: 11.33 years; range: $26-87$ years), 147 patients with AMI (48.5\%) were identified as having poor 
Table I Characteristics Of The Study Population According To Sleep Quality

\begin{tabular}{|l|l|l|l|}
\hline & $\begin{array}{l}\text { Poor Sleep } \\
\text { Quality } \\
\text { (n=I47) }\end{array}$ & $\begin{array}{l}\text { Good Sleep } \\
\text { Quality } \\
\text { (n=156) }\end{array}$ & P value \\
\hline Age (year) & $60.12(11.24)$ & $59.12(11.44)$ & 0.440 \\
\hline Male (\%) & 61.2 & 67.3 & 0.269 \\
\hline Hypertension (\%) & 50.7 & 47.2 & 0.547 \\
\hline Diabetes (\%) & 28.7 & 30.7 & 0.707 \\
\hline $\begin{array}{c}\text { Medication } \\
\beta \text {-blocker (\%) } \\
\text { ACE-I/ARB (\%) }\end{array}$ & 15.4 & 12.7 & 0.526 \\
Amiodarone (\%) & 2.2 & 16.0 & 0.766 \\
\hline Anterior AMI (\%) & 52.2 & 1.2 & 0.662 \\
\hline QTc (ms) & $446.85 \pm 33.18$ & $452.21 \pm 36.87$ & 0.197 \\
\hline EF (\%) & $59.0(8.4)$ & $61.0(7)$ & 0.271 \\
\hline Ischemic times (h) & $5.0(5.38)$ & $5.0(4.0)$ & 0.558 \\
\hline LVEDD (mm) & $45.6(5.63)$ & $45.2(5.4)$ & 0.996 \\
\hline
\end{tabular}

Notes: Data are presented as mean \pm s.d, medians with IQR or number (\%). The $\chi^{2}$ statistical test or Fisher's exact test for nominal variables and T or Mann-Whitney $U$-test for continuous variables were performed to assess whether there were significant differences between the groups with and without sleep disorder.

Abbreviations: ACE-I, angiotensin-converting-enzyme inhibitor; ARB, angiotensin receptor blocker; QTc, corrected QT intervals; EF, ejection fraction; LVEDD, left ventricular end-diastolic internal diameter; $\beta$-blocker, beta-receptor antagonist.

sleep quality and $71(23.4 \%)$ individuals were identified as suffering VT after AMI. Subjects with VT after AMI had a higher prevalence of poor sleep quality than those without VT after AMI (63.4\% vs 44.0\%; $\mathrm{P}<0.01)$. No significant differences in sleep quality were found in two groups on the basis of age, gender, hypertension, diabetes, and history of medication, location of AMI, EF, QTc, ischemic times, LVEDD.

The results of the multivariable logistic regression analysis about the associations between the global PSQI as well as its components and the prevalence of VT during the first week of AMI is shown in Table 2. After adjusting to age, gender, hypertension, diabetes, history of medication, location of AMI, EF, QTc, ischemic times, LVEDD, scores of global PSQI were found to be in relation to the prevalence of post-AMI VT. The odds of VT among subjects with a global PSQI score of 6 or more were 1.98 (95\% CI, 1.11-3.50) compared to those among subjects whose PSQI global scores were 5 or less. In addition, there were associations between prevalence of VT with four components of PSQI in subjects of both groups: subjective sleep quality, sleep duration, sleeps disturbance, and daytime dysfunction.

Time and frequency domain of HRV were analyzed in patients with AMI, as shown in Table 3. A statistically significant increase was found in AHR value in subjects with poor sleep quality compared to those with good sleep quality. There was a statistically significant difference in rMSSD value between two groups $(\mathrm{P}<0.05)$.

However, no statistical difference was found in SDNN, SDANN, SDANN index, pNN50 variables. Compared to participants with good sleep quality, those with poor sleep quality showed a significant increase in LF and reduction in $\mathrm{HF}(\mathrm{P}<0.05)$. However, there was no significant difference in VLF and ULF between these two groups. In addition, LF/ $\mathrm{HF}$ ratio was also significantly different in these two groups, indicating the presence of autonomic imbalance $(\mathrm{P}<0.05)$.

To further investigate the effect of sleep quality on catecholamine expression in patients with AMI, we measured the concentrations of adrenaline, norepinephrine and dopamine in a subgroup including 80 patients. No significant difference was found in the baseline demographic and clinical characteristics in both groups, as shown in Table 4. The plasma levels of adrenaline and norepinephrine in patients with poor sleep quality were higher than those with good sleep quality. However, no difference in plasma levels of dopamine between two groups was found.

Spearman correlations were determined among PSQI and values of adrenaline, norepinephrine, and dopamine (Table 5). There were significant direct correlations between PSQI with adrenaline and norepinephrine (adrenaline:rho $=0.39, \mathrm{P}<0.001$, norepinephrine:rho $=0.42$, $\mathrm{P}<0.001)$. The correlations between PSQI with dopamine did not be achieved (adrenaline: rho $=0.02, \mathrm{P}=0.91$ ).

\section{Discussion}

In contrast to the majority of previous studies, we took both sleep quantity and quality into consideration to assess the global sleep status in patients with AMI in the present study. We used PSQI to investigate the potential association of the global sleep quality with VT prevalence in AMI patients. Our results demonstrated that short-lasting poor sleep quality was associated with VT prevalence in patients in the first week of AMI. We also observed significant associations between the risk of VT after AMI and the components of PSQI scores: poor sleep quality, short sleep duration, sleep disturbance and daytime dysfunction. To date, few studies reported the effect of the global sleep status on HRV and catecholamine levels in patients with 
Table 2 Prevalence And Odds Ratio Of VT After AMI By PSQI And Its Components

\begin{tabular}{|c|c|c|c|c|c|c|}
\hline & Score & $\mathbf{n}$ & Prevalence \% & OR & $95 \% \mathrm{Cl}$ & $P$ value \\
\hline \multirow[t]{2}{*}{ Global PSQI score } & $\leq 5$ & 156 & 16.7 & 1.00 & (Reference) & \\
\hline & $\geq 6$ & 147 & 30.6 & 1.98 & $1.11-3.50$ & $<0.05$ \\
\hline \multirow[t]{4}{*}{ Subjective sleep quality } & 0 & 110 & 14.5 & & (Reference) & \\
\hline & I & 95 & 25.3 & 2.34 & $1.12-4.92$ & $<0.05$ \\
\hline & 2 & 54 & 29.6 & 2.52 & I.II-5.7| & $<0.05$ \\
\hline & 3 & 44 & 34.1 & 2.82 & $1.20-6.64$ & $<0.05$ \\
\hline \multirow[t]{4}{*}{ Sleep latency } & 0 & 132 & 18.2 & & (Reference) & \\
\hline & $\mathrm{I}$ & 96 & 25.0 & 1.45 & $0.74-2.80$ & 0.277 \\
\hline & 2 & 39 & 33.3 & 1.60 & $0.68-3.72$ & $0.28 I$ \\
\hline & 3 & 36 & 27.8 & 1.48 & $0.6 I-3.6 I$ & 0.388 \\
\hline \multirow[t]{4}{*}{ Sleep duration } & 0 & 111 & 13.5 & & (Reference) & \\
\hline & 1 & 83 & 26.5 & 2.58 & $1.20-5.55$ & $<0.05$ \\
\hline & 2 & 67 & 28.4 & 2.87 & $1.28-6.42$ & $<0.05$ \\
\hline & 3 & 42 & 35.7 & 3.34 & $1.40-7.95$ & $<0.01$ \\
\hline \multirow[t]{4}{*}{ Habitual sleep efficiency } & 0 & 130 & 16.9 & & (Reference) & \\
\hline & 1 & 76 & 26.3 & 1.67 & $0.8 \mathrm{I}-3.44$ & 0.169 \\
\hline & 2 & 54 & 29.6 & 1.92 & $0.88-4.17$ & 0.100 \\
\hline & 3 & 43 & 30.2 & 2.10 & $0.92-4.80$ & 0.079 \\
\hline \multirow[t]{4}{*}{ Sleep disturbance } & 0 & 125 & 14.4 & & (Reference) & \\
\hline & 1 & 106 & 25.5 & 2.12 & $1.06-4.22$ & $<0.05$ \\
\hline & 2 & 39 & 35.9 & 2.94 & $1.23-7.01$ & $<0.05$ \\
\hline & 3 & 33 & 36.4 & 3.28 & $1.32-9.10$ & $<0.05$ \\
\hline \multirow[t]{2}{*}{ Use of sleep medication } & 0 & 273 & 22.0 & & (Reference) & \\
\hline & $\geqslant 1$ & 30 & 36.7 & 2.05 & $0.88-4.77$ & 0.096 \\
\hline \multirow[t]{4}{*}{ Daytime dysfunction } & 0 & 147 & 14.3 & & (Reference) & \\
\hline & 1 & 78 & 30.8 & 2.63 & $1.30-5.33$ & $<0.01$ \\
\hline & 2 & 47 & 31.9 & 2.91 & $1.29-6.57$ & $<0.05$ \\
\hline & 3 & 31 & 35.5 & 3.17 & $|.29-7.8|$ & $<0.05$ \\
\hline
\end{tabular}

Notes: Data are presented as adjusted odds ratio ( $95 \%$ confidence interval) for the prevalence of VT after AMI. Logistic regression analysis was conducted after adjusted for age, gender, hypertension, diabetes, history of medication, location of AMI, EF, QTc, ischemic times, LVEDD.

Abbreviations: $\mathrm{Cl}$, confidence interval; OR, odds ratio; PSQI, Pittsburgh sleep quality index.

AMI. Our study indicated that the AMI patients with SDs showed a significant increase in LF as well as LF/HF ratio and reduction in HF, suggesting sympathetic hyperactivity. In addition, higher levels of adrenaline and norepinephrine in patients with poor sleep quality were found than those in patients with good sleep quality.

Previous studies have shown that sleep quality is associated with increased risk of VT. Animal experiments indicated that sleep deprivation increases the latency times of lethal VA in rat. ${ }^{12}$ Similarly, significantly higher proportions of arrhythmia, including VT, were found in community elderly with insomnia than those without insomnia in China. ${ }^{13}$ In a recent clinical study, men who reported sometimes sleep disturbances within 4 weeks before AMI showed an increased mortality risk compared with those without complaints in a model adjusted by confounding variables. ${ }^{14}$ It is generally accepted that VT/VF are the most common causes of sudden death in patients with AMI. ${ }^{15}$ Thus, it was suggested that there may be a link between SDs and VT risk in patients with AMI. In our study, there is a significant relation with poor sleep quality and the prevalence of VT in patients with AMI. Consistently, it has been found that central sleep apnea is significantly linked with VT/VF in chronic heart failure, which is the most frequent cause of sudden cardiac death in those patients. ${ }^{16,17}$ In addition, SDs have been shown to cause the worsening of clinical outcomes in AMI patients. $^{18}$ These results suggest that SD is one of the 
Table 3 Heart Rate Variability Indices Of Participants Grouped According To Their Sleep Quality

\begin{tabular}{|l|l|l|l|}
\hline Parameters & $\begin{array}{l}\text { Poor Sleep } \\
\text { Quality } \\
\text { (n=I47) }\end{array}$ & $\begin{array}{l}\text { Good Sleep } \\
\text { Quality } \\
\text { (n=156) }\end{array}$ & P value \\
\hline AHR (beats/min) & $75.08 \pm 13.56$ & $72.50 \pm 10.52$ & $<0.05$ \\
SDNN (In ms) & $4.36 \pm 0.35$ & $4.39 \pm 0.35$ & 0.529 \\
SDANN (In ms) & $4.18 \pm 0.38$ & $4.22 \pm 0.38$ & 0.342 \\
rMSDD (In ms) & $2.97 \pm 0.59$ & $3.10 \pm 0.52$ & $<0.05$ \\
SDNNi ms & $40.0(23)$ & $43.5(21)$ & 0.290 \\
PNN 50\% & $1.0(4)$ & $1.5(4)$ & 0.150 \\
TP (In ms $\left.{ }^{2}\right)$ & $9.89 \pm 0.82$ & $10.00 \pm 0.74$ & 0.175 \\
HF (In ms $\left.{ }^{2}\right)$ & $5.33 \pm 1.10$ & $5.58 \pm 1.06$ & 0.050 \\
LF (In ms $\left.{ }^{2}\right)$ & $6.90 \pm 0.76$ & $6.66 \pm 0.83$ & $<0.01$ \\
LF/HF (In) & $1.43 \pm 0.83$ & $1.14 \pm 0.75$ & $<0.01$ \\
vLF (In ms $\left.{ }^{2}\right)$ & $8.06 \pm 0.83$ & $8.22 \pm 0.82$ & 0.103 \\
uLF (In ms $\left.{ }^{2}\right)$ & $9.67 \pm 0.88$ & $9.72 \pm 0.83$ & 0.574 \\
\hline
\end{tabular}

Notes: Data are presented as mean \pm s.d, medians with IQR or number (\%). The $\chi^{2}$ statistical test for nominal variables and T or Mann-Whitney U-test for continuous variables were performed.

Abbreviations: AHR, $24 \mathrm{hr}$ average heart rates; SDNN, the standard deviation of mean NN; standard deviation of all RR intervals; SDANN, the standard deviation of all 5 min mean RR intervals; rMSSD, root-mean-square of the successive RR interval difference; SDNNi, the mean of the standard deviation of all normal RR intervals for all 5-min segments; pNN50, the percentage of $>50$ ms differences between adjacent $\mathrm{NN}$; TP, total power (sum of VLF, LF and HF); LF, low frequency power; HP, high frequency power; vLF, very low frequency power; uLF, ultra-low-frequency; LF/HF, the ratio of low to high frequency power; In, natural logarithm.

important factors affecting the prognosis of patients with AMI and the routine evaluation of sleep quality in patients with AMI should not be neglected.

There is a growing interest concerning about HRV, mainly due to its possible association with sleep quality and cardiovascular diseases. Current evidences suggest that HRV is adversely affected by poor sleep quality and short sleep duration. ${ }^{19-21}$ Studies convinced significantly higher LF and LF/HF, and lower HF in subjects with OSA or sleep deprivation. ${ }^{22,23}$ We found that poor sleep quality significantly related to reduced HRV in patients with AMI. Similarly, studies in rats have shown that there is higher frequency of sleep interruption and higher LF and LF/HF ratio in the MI group than those in the sham group. ${ }^{24,25}$ These results suggest that SD results in impairment of HRV in AMI patients. Convincing evidences demonstrated that impaired HRV plays an important role in the prediction of acute myocardial infarction, arrhythmias and sudden cardiac death. ${ }^{26,27}$ Furthermore, increased LF/HF ratio and higher LF have been found to reflect increased sympathetic activity and decreased parasympathetic activity. These evidences support the hypothesis that sleep-related reduction of HRV in AMI patients is significantly associated with hyperactivation of the sympathetic nervous system.
Table 4 Baseline Characteristics And The Plasma Level Of Catecholamine Of The Study Population

\begin{tabular}{|l|l|l|l|}
\hline Overall (n=80) & $\begin{array}{l}\text { Poor Sleep } \\
\text { Quality (n=40) }\end{array}$ & $\begin{array}{l}\text { Good Sleep } \\
\text { Quality (n=40) }\end{array}$ & P value \\
\hline Age (year) & $57.68 \pm 12.57$ & $54.93 \pm 10.40$ & 0.290 \\
\hline Male (\%) & 65.0 & 62.5 & 0.816 \\
\hline Hypertension (\%) & 50.0 & 55.0 & 0.639 \\
\hline Diabetes (\%) & 37.5 & 32.5 & 0.707 \\
\hline $\begin{array}{c}\text { Medication } \\
\text { 3-blocker (\%) }\end{array}$ & $15.0 \%$ & $17.5 \%$ & 0.762 \\
ACE-I /ARB (\%) & $17.5 \%$ & $20.5 \%$ & 0.766 \\
Amiodarone (\%) & $2.5 \%$ & $5.0 \%$ & 1.000 \\
\hline Anterior AMI (\%) & 45.0 & 40.0 & 0.651 \\
\hline EF (\%) & $60.0(3.75)$ & $60.5(6.75)$ & 0.908 \\
\hline ischemic times (h) & $3.5(3.0)$ & $4.0(4.0)$ & 0.577 \\
\hline LVEDD (mm) & $44.6(5.17)$ & $43.8(5.92)$ & $0.88 I$ \\
\hline NE (nmol/L) & $2.78 \pm 0.73$ & $2.31 \pm 0.89$ & $<0.05$ \\
\hline E (nmol/L) & $0.29 \pm 0.15$ & $0.22 \pm 0.14$ & $<0.05$ \\
\hline DA (nmol/L) & $0.37 \pm 0.18$ & $0.40 \pm 0.23$ & 0.518 \\
\hline Notes Dam a pra & & & \\
\hline
\end{tabular}

Notes: Data are presented as mean \pm s.d, median (quartile spacing) or number (\%). The $\chi^{2}$ statistical test or Fisher's exact test for nominal variables and Tor MannWhitney U-test for continuous variables were performed to assess whether there were significant differences between the groups with and without sleep disorder. Abbreviations: ACE-l,angiotensin-converting-enzyme inhibitor; ARB angiotensin receptor blocker; $\beta$-blocker, beta-receptor antagonist; QTc, Corrected QT intervals; EF, ejection fraction; LVEDD, left ventricular end-diastolic internal diameter; $\mathrm{NE}$, norepinephrine; E, adrenaline; DA, dopamine.

Table 5 The Coefficient Of Correlation Between The Plasma Level Of Catecholamine With PSQI

\begin{tabular}{|l|l|l|l|}
\hline & PSQI & N & P value \\
\hline NE $(\mathrm{nmol} / \mathrm{L})$ & 0.422 & 80 & $<0.001$ \\
E $(\mathrm{nmol} / \mathrm{L})$ & 0.388 & 80 & $<0.001$ \\
DA $(\mathrm{nmol} / \mathrm{L})$ & 0.018 & 80 & 0.913 \\
\hline
\end{tabular}

Notes: A rank correlation analysis was conducted for the relation between the plasma level of catecholamine with PSQI.

Abbreviations: NE, norepinephrine; E, adrenaline; DA dopamine; PSQI, Pittsburgh sleep quality index.

In order to detect the effects of poor sleep on sympathetic nerve activity, we measured the concentration of catecholamine in patients with AMI. Our study indicated that the concentration of adrenaline in the group with poor sleep quality was significantly higher than that in the control group. This suggests that sympathetic activity may be further activated in patients with AMI when their sleep quality decreases. Consistent with our study, previous study has 
reported that sympathetic nerve activity increases in patients with myocardial infarction and sleep apnea. ${ }^{28}$ Human and animal data have shown that increased sympathetic tone of heart predisposes post-AMI patients to abnormal cardiac electrical activity. For instance, electrical storms, which are characterized by the excessive activation of sympathetic activity, contribute to the prevalence of recurrent ventricular arrhythmias after AMI. ${ }^{29}$ On the contrary, sympathetic blockade, either in the form of beta-receptor or left stellate ganglionic blockade, is known to be highly effective in preventing the onset of VA following AMI in animal and clinical trials. ${ }^{30,31}$ Furthermore, in the presence of cardiac ischemia, sympathetic stimulation can facilitate the initiation of VT by inducing changes in ECG repolarization and reducing fibrillation threshold, as shown by experimental data. ${ }^{32}$ On the basis of these observations, it is biologically plausible that SDs may trigger VT in patients with AMI by increasing sympathetic tone.

The current study has some limitations. First, any causal relationship cannot be demonstrated between VT and sleep status, due to the nature of the cross-sectional study. Secondly, sleep quality was evaluated by self-reported brief questionnaires in our study. Sleep evaluation based on of polysomnography might be more objective. However, polysomnography is not the most infeasible monitoring tool for a large number of people with severe cardiovascular diseases. Thirdly, because presence of obstructive sleep apnea in these subjects was not investigated, we cannot exclude the potential influence of hypoxia on sleep disorders and post-AMI VT as well as stress hormone secretion. Fourthly, the limitation of rank correlation analysis made it impossible to examine any potential influence of many factors on the association between PSQI scores and plasma levels of catecholamine. Therefore, the observed relationship between sleep quality and sympathetic activity should be viewed with caution. Finally, the self-report sleep measures were subject to recall bias, because participants' sleep habits over the past month and week were reported by their memories.

\section{Conclusion}

Sleep status was significantly associated with the occurrence of VT within the first week of AMI. The effect of poor sleep quality on sympathetic nerve activity may contribute to the prevalence of VT following AMI. Based on these findings, it is essential to evaluate sleep quality for patients with AMI as a routine, as timely improvment of the identified sleep problems including usage of drugs for sleep may prevent the prevalence of VT after AMI. Furthermore, more attention should be paid to sympathetic nerve activity in AMI patients with sleep disorders and adequate use of anti-sympathetic drugs may be an effective strategy to improve the prognosis of myocardial infarction.

\section{Ethics Approval}

This cross-sectional study was approved by the Ethics Committee of The Second Affiliated Hospital of Harbin Medical University (ky2018-246) and all experimental procedures conformed to the ethical guidelines of the 1975 Declaration of Helsinki. All patients signed the written informed consent documents.

\section{Abbreviations}

VT, ventricular tachycardia; AMI, acute myocardial infarction; SDs, sleep disorders; VAs, ventricular arrhythmias; PSQI, Pittsburgh Sleep Quality Index; Holter, 24-h ambulatory electrocardiography; QTc, corrected QT intervals; HRV, heart rate variability; STEMI, ST-segment elevation myocardial infarction; PCI, percutaneous coronary intervention; ECG, electrocardiogram; EF, ejection fraction; LVEDD, left ventricular end-diastolic internal diameter; AHR, $24 \mathrm{hr}$ average heart rates; SDNN, the standard deviation of mean NN; standard deviation of all RR intervals; SDANN, the standard deviation of all $5 \mathrm{~min}$ mean RR intervals; rMSSD, root-mean-square of the successive RR interval difference; SDNNi, the mean of the standard deviation of all normal RR intervals for all 5-min segments; pNN50, the percentage of $>50 \mathrm{~ms}$ differences between adjacent NN; TP, total power (sum of VLF, LF and HF); LF, low frequency power; HP, low frequency power; vLF, very low frequency power; uLF, ultra-lowfrequency; $\mathrm{LF} / \mathrm{HF}$, the ratio of low to high frequency power; EDTA, ethylenediaminetetraacetic acid; IQR, interquartile range; $\mathrm{CI}$, confidence interval.

\section{Acknowledgments}

We are very grateful to Dr. Pengzhou Hang for his research assistance.

\section{Funding}

This research was supported by grants from the Health Commission Foundation of Heilongjiang Province (2018065) and the Heilongjiang Province Postdoctoral Science Foundation (LBH-Z18212).

\section{Disclosure}

The authors declare no conflict of interest. 


\section{References}

1. Mont L, Cinca J, Blanch P, et al. Predisposing factors and prognostic value of sustained monomorphic ventricular tachycardia in the early phase of acute myocardial infarction. J Am Coll Cardiol. 1996;28 (7):1670-1676.

2. Bailey JJ, Berson AS, Handelsman H, Hodges M. Utility of current risk stratification tests for predicting major arrhythmic events after myocardial infarction. J Am Coll Cardiol. 2001;38(7):1902-1911. doi:10.1016/s0735-1097(01)01667-9

3. Leger D, Poursain B, Neubauer D, Uchiyama M. An international survey of sleeping problems in the general population. Curr Med Res Opin. 2008;24(1):307-317. doi:10.1185/030079907x253771

4. Ren Y, Liu Y, Meng T, et al. Social-biological influences on sleep duration among adult residents of Northeastern China. Health Qual Life Outcomes. 2019;17(1):47. doi:10.1186/s12955-019-1111-3

5. Liu RQ, Qian Z, Trevathan E, et al. Poor sleep quality associated with high risk of hypertension and elevated blood pressure in China: results from a large population-based study. Hypertens Res. 2016;39 (1):54-59. doi:10.1038/hr.2015.98

6. Taylor DJ, Mallory LJ, Lichstein KL, Durrence HH, Riedel BW, Bush AJ. Comorbidity of chronic insomnia with medical problems. Sleep. 2007;30(2):213-218. doi:10.1093/sleep/30.2.213

7. Redline S, Foody J. Sleep disturbances: time to join the top 10 potentially modifiable cardiovascular risk factors? Circulation. 2011;124 (19):2049-2051. doi:10.1161/CIRCULATIONAHA.111.062190

8. van Amelsvoort LG, Schouten EG, Maan AC, Swenne CA, Kok FJ. Changes in frequency of premature complexes and heart rate variability related to shift work. Occup Environ Med. 2001;58(10):678681. doi:10.1136/oem.58.10.678

9. Akhil R, Ryan C, Anusha K, Tereshchenko LG, Akram K. A systematic review of the association between obstructive sleep apnea and ventricular arrhythmias. J Clin Sleep Med. 2014;10(10):11551160. doi: $10.5664 / \mathrm{jcsm} .4126$

10. Warner MR, Kroeker TS, Zipes DP. Sympathetic stimulation and norepinephrine infusion modulate extracellular potassium concentration during acute myocardial ischemia. Circ Res. 1992;71(5):10781087. doi:10.1161/01.res.71.5.1078

11. Westermann J, Hubl W, Kaiser N, Salewski L. Simple, rapid and sensitive determination of epinephrine and norepinephrine in urine and plasma by non-competitive enzyme immunoassay, compared with HPLC method. Clin Lab. 2002;48(1-2):61-71.

12. Joukar S, Ghorbani-Shahrbabaki S, Hajali V, Sheibani V, Naghsh N. Susceptibility to life-threatening ventricular arrhythmias in an animal model of paradoxical sleep deprivation. Sleep Med. 2013;14 (12):1277-1282. doi:10.1016/j.sleep.2013.07.008

13. Wang YM, Song M, Wang R, et al. Insomnia and multimorbidity in the community elderly in China. J Clin Sleep Med. 2017;13(4):591597. doi: $10.5664 /$ jesm. 6550

14. Nairz F, Meisinger C, Kirchberger I, et al. Association of sleep disturbances within 4 weeks prior to incident acute myocardial infarction and long-term survival in male and female patients: an observational study from the MONICA/KORA myocardial infarction registry. $B M C$ Cardiovasc Disord. 2018;18(235). doi:10.1186/s12872-12018-10969-z

15. Barger LK, Rajaratnam SMW, Cannon CP, et al. Short sleep duration, obstructive sleep apnea, shiftwork, and the risk of adverse cardiovascular events in patients after an acute coronary syndrome. J Am Heart Assoc. 2017;6(10):e006959. doi:10.1161/JAHA.117.006959

16. Grimm W, Koehler U. Cardiac arrhythmias and sleep-disordered breathing in patients with heart failure. Int J Mol Sci. 2014;15 (10):18693-18705. doi:10.3390/ijms151018693
17. Saman N. Ventricular arrhythmia and sleep-related breathing disorders. Heart Rhythm. 2011;8(5):663-664. doi:10.1016/j.hrthm.2010.12.043

18. Andrechuk CR, Ceolim MF. Sleep quality and adverse outcomes for patients with acute myocardial infarction. J Clin Nurs. 2016;25(12):223-230. doi:10.1111/jocn.13051

19. Chung MH, Kuo TB, Hsu N, Chu H, Chou KR, Yang CC. Sleep and autonomic nervous system changes - enhanced cardiac sympathetic modulations during sleep in permanent night shift nurses. Scand J Work Environ Health. 2009;35(3):180-187. doi:10.5271/ sjweh.1324

20. Nielsen T, Paquette T, Solomonova E, Lara-Carrasco J, Colombo R, Lanfranchi P. Changes in cardiac variability after REM sleep deprivation in recurrent nightmares. Sleep. 2010;33(1):113-122. doi:10.1093/sleep/ 33.1.113

21. Zhong X, Hilton HJ, Gates GJ, et al. Increased sympathetic and decreased parasympathetic cardiovascular modulation in normal humans with acute sleep deprivation. J Appl Physiol. 2005;98 (6):2024-2032. doi:10.1152/japplphysiol.00620.2004

22. Kesek M, Franklin KA, Sahlin C, Lindberg E. Heart rate variability during sleep and sleep apnoea in a population based study of 387 women. Clin Physiol Funct Imaging. 2009;29(4):309-315. doi:10.1111/j.1475-097X. 2009.00873.x

23. Narkiewicz K, Somers VK. Sympathetic nerve activity in obstructive sleep apnoea. Acta Physiol Scand. 2003;177(3):385-390. doi:10.1046/ j.1365-201X.2003.01091.x

24. Lin WL, Chen YR, Lai CT, et al. Neural mechanism of angiotensinconverting enzyme inhibitors in improving heart rate variability and sleep disturbance after myocardial infarction. Sleep Med. 2018;48:61-69. doi:10.1016/j.sleep.2018.04.007

25. Lin WL, Lo LW, Chen HR, et al. Sleep-related changes in cardiovascular autonomic regulation in left coronary artery ligation rats: neural mechanism facilitating arrhythmia after myocardial infarction. Int J Cardiol. 2016;225:65-72. doi:10.1016/j.ijcard.2016.09. 121

26. Kleiger RE, Miller JP, Bigger JT Jr., Moss AJ. Decreased heart rate variability and its association with increased mortality after acute myocardial infarction. Am J Cardiol. 1987;59(4):256-262. doi:10. 1016/0002-9149(87)90795-8

27. Makikallio TH, Barthel P, Schneider R, et al. Prediction of sudden cardiac death after acute myocardial infarction: role of Holter monitoring in the modern treatment era. Eur Heart J. 2005;26(8):762769. doi:10.1093/eurheartj/ehi188

28. Yamashita J, Nomura M, Uehara K, et al. Influence of sleep apnea on autonomic nervous activity and QT dispersion in patients with essential hypertension and old myocardial infarction. $J$ Electrocardiol. 2004;37(1):31-40. doi:10.1016/j.jelectrocard.2003. 10.009

29. Vaseghi M, Gima J, Kanaan C, et al. Cardiac sympathetic denervation in patients with refractory ventricular arrhythmias or electrical storm: intermediate and long-term follow-up. Heart Rhythm. 2014;11 (3):360-366. doi:10.1016/j.hrthm.2013.11.028

30. Nademanee K, Taylor R, Bailey WE, Rieders DE, Kosar EM. Treating electrical storm : sympathetic blockade versus advanced cardiac life support-guided therapy. Circulation. 2000;102(7):742747. doi:10.1161/01.cir.102.7.742

31. Anderson JL, Rodier HE, Green LS. Comparative effects of betaadrenergic blocking drugs on experimental ventricular fibrillation threshold. Am J Cardiol. 1983;51(7):1196-1202. doi:10.1016/00029149(83)90368-5

32. Opthof T, Misier AR, Coronel R, et al. Dispersion of refractoriness in canine ventricular myocardium. Effects of sympathetic stimulation. Circ Res. 1991;68(5):1204-1215. doi:10.1161/01.res.68.5.1204 


\section{Publish your work in this journal}

Nature and Science of Sleep is an international, peer-reviewed, open access journal covering all aspects of sleep science and sleep medicine, including the neurophysiology and functions of sleep, the genetics of sleep, sleep and society, biological rhythms, dreaming, sleep disorders and therapy, and strategies to optimize healthy sleep.
The manuscript management system is completely online and includes a very quick and fair peer-review system, which is all easy to use. Visit http://www.dovepress.com/testimonials.php to read real quotes from published authors. 